\title{
Geometric features of LOS data derived by SAR PSI technologies and the three- dimensional data fusion
}

László Bányai, Eszter Szűcs, Viktor Wesztergom

Geodetic and Geophysical Institute of the Research Centre for Astronomy and Earth Sciences, Hungarian Academy of Sciences, Csatkai E. u. 6-8., H-9400 Sopron, Hungary

\begin{abstract}
At present, the satellite SAR Persistent Scatterer Interferometry (PSI) can already estimate surface changes with a near to $1 \mathrm{~mm}$ theoretical precision limit. However, the ascending and descending acquisitions of available SAR services cannot provide three-dimensional changes routinely, though the slow deformation processes are basically three-dimensional (3D).

In this paper the geometric features of ascending and descending SAR data and possible fusion with geodetic data are summarised. All the geometric equations are introduced, which are necessary to derive the two characteristic changes in the observation plain defined by ascending and descending unit vectors pointing to SAR satellite positions. The unambiguously derivable characteristic changes can be transformed into vertical and east changes, but they may be biased by possible north displacement. The geometric features of symmetric and asymmetric acquisitions are also investigated. Monte-Carlo simulation is used to investigate the precision of two estimated components. It is experienced that the precisions are not sensitive to one degree standard deviations of positional angles.

The Gauss-Markov model of least square adjustment method is used to derive only the statistical properties of reasonable data fusion which can contribute to the $3 \mathrm{D}$ applications. Although complementary satellites, which are already proposed in the literature, could
\end{abstract}


provide precise autonomous solutions, in the practise GNSS and levelling data can be used for direct data fusion. Whereas, even errorless levelled high changes cannot contribute to the proper estimation of northern components, GNSS derived changes are the best candidates, which can be interpolated or measured directly. Moreover, these two techniques can properly compensate the weaknesses of each other. The interferometric SAR techniques are not sensitive enough to the north changes, but can contribute the precision of height estimation, which are the weakest components of the GNSS technique. This statement is valid if the standard deviations of combined data are comparable.

For test computations the geometric parameters of available Sentinel-1A images are used, which cover the area of the Széchenyi István Geophysical Observatory, where experimental integrated geodetic benchmark is located combining ascending and descending backscatterers with the possibility of the GNSS, gravimetric and traditional geodetic measurements, as well.

Keywords - InSAR, Persistent Scatter Interferometry, GNSS, levelling, data fusion, least square adjustment, Gauss-Markov model.

\section{Introduction}

The well-known satellite Synthetic Aperture Radar (SAR) technology is regularly used for the estimation of displacements and volume changes of the Earth surface by means of remote sensing and differential interferometric methods. There are plenty of reviews dealing with three-dimensional (3D) investigations (e.g. in Tralli et al., 2005 and Hu et al., 2014).

According to the theoretical and practical advances (Colesanti et al., 2003; Hooper, 2008; Hooper et al., 2012; Plank et al., 2013; Wasowski and Bovenga, 2014), the Persistent Scatter Interferometry (PSI) can provide surface change time series in LOS directions at a mm-cm precision level. In the best cases the precision is near to the $1 \mathrm{~mm}$ theoretical limit. This 
accuracy is already competitive compared to the high precision Global Navigation Satellite Systems (GNSS) and traditional geodetic techniques. The 3D absolute stereo localisation of persistent scatterers may be a successful alternative in the near future, using high resolution $\mathrm{X}$ band data (Gisinger at al. 2015). However, it is emphasized that this paper concentrates only on interferometric technologies, which can be optimally applied using Santinel-1 images freely available to the scientific community.

Moreover, at this level it is more reasonable to speak about the cooperation than a competition, if the advantages and disadvantages of different techniques are taken into account.

Those geoscientists who deal with the slow deformation of the earth surfaces are basically interested in the estimation of 3D displacement fields. Though GNSS and geodetic techniques provide 3D displacements, the maintenance of the continuous networks or the repeated measurements of epoch networks are relatively expensive and provide usually sparse data distribution in space or time, respectively.

The success of PSI technology strongly depends on the recognition of large numbers of accurate persistent scatterers (PS) in coherent SAR images (Riddick et al., 2012; Garthwaite et al., 2013). The territorial coverage of PSs may be more favourable than the benchmarks of GNSS networks, but the resolution cells (or pixels) cover areas of several square meters and additionally the LOS changes are referred to only one spatial direction.

There are several excellent practical applications where ascending or/and descending LOS data are combined with theoretical models (e.g. glaciers, volcanos, landslides or earthquakes) of expected surface changes, e.g. Wright et al., (2004); Hooper et al., (2004); Cascini et al., (2010); Meyer et al., (2015); Kumar et al., (2011). They strongly depend on the investigated phenomenon and their proper preliminary models; therefore they are not investigated in this paper. 
If satellite missions can provide LOS data of same PSs during ascending and descending illuminations, two displacement components can be estimated. The vertical (or up) and east components can be derived if the north components are negligible (Manzo et al., 2006), otherwise the estimated vertical displacements are biased by north movements.

From a theoretical point of view, at least three or more ascending and descending LOS observations can provide direct 3D displacements. A successful case study was presented in Gray (2006) using RADARSAT data, but the procedure can be applied effectively only in high latitude regions, where the positional directions are sufficiently different. The direct 3D applications have already been discussed in the literature (Wright et al., 2004; Rocca, 2003) supposing the combinations of usual right and/or possible left looking acquisitions. In Wright et al. (2004) an additional SAR mission is proposed with a satellite inclination of $60^{\circ} / 120^{\circ}$ as one of the proper solutions, which covers the Earth between $60^{\circ} \mathrm{S}$ and $60^{\circ} \mathrm{N}$ latitudes. In the lack of such missions the combination of different data sources are preferred.

The common application of dense PSs and sparse 3D GNSS (or traditional) and 1D levelling data require the proper areal overlapping of the different networks. Another requirement is the proper interpolation of 3D and/or 1D geodetic data to the position of PSs cells. The interpolations can be carried out by using different deterministic models or stochastic methods. In Hung et al. (2011) the combination of SAR and levelling data is demonstrated for subsidence investigation on a large area, using kriging and draping method. The levelling data can improve the subsidence estimation (e.g. reducing the bias caused by possible north movements) but cannot help the estimation of 3D displacements.

While there will be no complementary SAR satellite missions at our disposal or the stereo localization cannot be applied routinely, thus autonomous 3D displacement estimation is not possible by this way; therefore the data fusion with GNSS displacements is the best candidate for the combinations. Excellent applications and methods are presented in several papers 
(Gudmundsson et al., 2002; Samsonov et al., 2007; Guglielmino et al., 2011; Catalao et al., 2011). Though the interpolation errors of GNSS derived changes effect the accuracy of data combination, they effectively compensate the weaknesses of each other.

The high accuracy of recent PSI technologies and the ESA Sentinel-1 C band mission, which is very near to the fully operational phase, can significantly raise the number of practical investigations, since the Sentinel-1A satellite (repeat cycle 12 days), and later in tandem mode with Sentinel-1B will be regularly available for the end-users free of charge.

The collocated or integrated application of artificial ascending and descending backscatterers and multi-purpose geodetic/geodynamics benchmark can open a new area of combined applications. It can be used in areas covered by vegetation, where the PSI technology cannot be applied routinely, or the properly installed integrated benchmark can guarantee the common geometric reference of the different sources of data. It can be utilized at hazardous areas or can be used for stability monitoring of those fundamental geodetic benchmarks, which are used to maintain the terrestrial reference frames for industrial and/or scientific purposes.

In this manuscript we concentrate only on the application of PSI derived ascending and descending data and the possible geodetic data combination to provide high precision 3D displacement estimations, regardless of the fact that the geodetic data are interpolated or directly measured.

In Section 2.1 the information content and the features of ascending and descending LOS data are discussed by geometric and algebraic methods. In Section 2.2 the usual direct data fusion and their precisions are investigated by the Gauss-Markov model of the least square adjustment method, which is frequently used in geodetic and surveying applications.

Although, the design of integrated benchmarks is not subject of this manuscript, the geometric parameters of the practical investigations are taken from the position of experimental 
integrated benchmark (http://www.ggki.hu/nc/en/news/) located in Széchenyi István Geophysical Observatory and Sentinel-1A orbit data of single look complex (SLC) images acquired from 03.10.2014. Images of two ascending and one descending frames are chosen. Since the high precision applications are addressed, the measurements are treated by $\sigma=2$ mm standard deviation (precision), which can be provided by precise GNSS and geodetic measurement together with properly designed artificial backscatterers if coherent SAR images are available.

\section{Theoretical background}

During the derivation of algebraic equations displacements between two epochs of PSI time series are chosen. These equations are valid if the displacements are replaced by average velocities estimated for longer time period. Since the same geometric reference system is used and the same reference areas can be chosen in overlapping ascending and descending images (see StaMPS/MTI manual, Hooper et al., 2012), common PSs can be combined.

\subsection{Geometric features of ascending and descending LOS data}

The geometry of ascending and descending observations referring to same PS (or more generally to same resolution cell on the Earth surface) is presented in Fig. 1, where $\alpha_{a}$ and $\alpha_{d}$ are the LOS azimuths, $\theta_{a}$ and $\theta_{d}$ are the incidence (or zenith) angles of the right looking illuminations, $\boldsymbol{s}_{a}$ and $\boldsymbol{s}_{d}$ are the unit vectors of ascending and descending satellite directions, all given in the ellipsoidal topocentric coordinate system, which is attached to PS at the reference epoch (or on master image), $\mathrm{U}$ is the direction of ellipsoidal normal (up), $\mathrm{N}$ is a direction to the north and $\mathrm{E}$ is the direction to the east in the plane perpendicular to $\mathrm{U}$. The azimuths and incidence angles are changing slowly from PS to PS. 
According to the looking directions of the antennae the LOS azimuths are perpendicular to the azimuths of the satellite motions. During the satellite motion the backscattered signals are projected into the LOS directions and the distances of reflecting resolution cells are estimated. Using the phase values of master and slave images, the differential SAR interferometry (DInSAR, PSI) can determine the change of distances with high precision. In the SAR publications usually the PS to satellite azimuth is preferred to describe the changes, here for geometric convenience the LOS azimuths are chosen, which connects the satellite to the PS.

If the investigated PS is displaced with respect to the reference epoch and reference PS (on the slave images), the LOS changes can be derived from the known 3D coordinate changes according to Fig. 2 using the known method of coordinate transformation. In Fig. 2a the displaced PS is shown in a 3D local system, Fig. 2b depicts the impact of the horizontal components $(\Delta E$ and $\Delta N)$ and Fig. 2c contains the additional impact of $\Delta U$ component. According to Fig. $2 b$ the effects of the horizontal components are

$$
\begin{aligned}
& \Delta R=\Delta E \sin \alpha+\Delta N \cos \alpha, \\
& \Delta A=\Delta E \cos \alpha-\Delta N \sin \alpha,
\end{aligned}
$$

where $\Delta R$ is a displacement along the LOS azimuth, while the perpendicular displacement $\Delta A$ cannot be sensed from this direction. According to Fig. 2c the additional impact of the vertical components is given by

$$
\begin{aligned}
& \Delta S=\Delta R \sin \theta-\Delta U \cos \theta, \\
& \Delta L=\Delta R \cos \theta+\Delta U \sin \theta,
\end{aligned}
$$

where $\Delta S$ is a displacement in LOS direction, while the perpendicular displacement $\Delta L$ cannot be sensed from this direction. If we accept the rule of thumb that the displacement should be positive if the PS moves toward the satellite, the sign of Eq. (3) has to be changed (Figs. 1 and 2), which leads to the basic equation

$$
\Delta S(\alpha, \theta)=\Delta U \cos \theta-(\Delta E \sin \alpha+\Delta N \cos \alpha) \sin \theta
$$


It is supposed that the differential InSAR or PSI data processing provides the time series of $\alpha_{a}, \theta_{a}, \Delta S_{a}$ and $\alpha_{d}, \theta_{d}, \Delta S_{d}$ values of the same PSs (or nearly the same resolution cells) for both ascending $(a)$ and descending $(d)$ satellites, the error sources are properly handled and the data refer to the same reference PS.

The measured changes (Eq. 5) contain information on all the three displacement components, but two measurements are not enough to directly determine three unknowns. Moreover, the unit vectors pointing to the two satellite positions define an observation plane in which two characteristic directions, I (inclination) and D (declination), can be identified. The intersection of this observation and N-E planes defines the axis D, while the axis I in the observation plane is perpendicular to axis D. The most important quantities that can be derived are summarised in Fig. 3.

The unit vectors $\left(\boldsymbol{s}_{a}, \boldsymbol{s}_{d}\right)$ can be determined according to Fig. 1, substituting the proper angles

$$
\left[\begin{array}{l}
S_{\mathrm{E}} \\
S_{\mathrm{N}} \\
S_{\mathrm{U}}
\end{array}\right]=\left[\begin{array}{c}
-\sin \alpha \sin \theta \\
-\cos \alpha \sin \theta \\
\cos \theta
\end{array}\right] .
$$

The vectorial multiplication

$$
\boldsymbol{n}=\boldsymbol{s}_{a} \times \boldsymbol{s}_{d}=\left[\begin{array}{c}
n_{\mathrm{E}} \\
n_{\mathrm{N}} \\
n_{\mathrm{U}}
\end{array}\right]
$$

provides the vector perpendicular to the plane. Because $\boldsymbol{s}_{a}$ and $\boldsymbol{s}_{d}$ are unit vectors

$$
\delta=\sin ^{-1} n
$$

holds, where $n$ is the length of the vector and $\delta$ is the angle between $\boldsymbol{s}_{a}$ and $\boldsymbol{s}_{d}$. If the vector components of $\boldsymbol{n}$ are divided by its length

$$
\boldsymbol{m}=\left[\begin{array}{l}
n_{\mathrm{E}} / n \\
n_{\mathrm{N}} / n \\
n_{\mathrm{U}} / n
\end{array}\right]=\left[\begin{array}{l}
m_{\mathrm{E}} \\
m_{\mathrm{N}} \\
m_{\mathrm{U}}
\end{array}\right]
$$

becomes a unit vector, too. According to the geometric background the following quantities can be derived 


$$
\begin{gathered}
\chi=\tan ^{-1}\left(m_{\mathrm{E}} / \sqrt{m_{\mathrm{E}}^{2}+m_{\mathrm{N}}^{2}}\right), \\
\alpha_{\mathrm{D}}=\frac{\pi}{2}+\chi, \\
\alpha_{\mathrm{I}}=\pi+\chi, \\
\boldsymbol{\omega}=\tan ^{-1}\left(m_{\mathrm{U}} / \sqrt{m_{\mathrm{E}}^{2}+m_{\mathrm{N}}^{2}}\right), \\
\boldsymbol{s}=\left[\begin{array}{l}
-m_{\mathrm{U}} \sin \chi \\
-m_{\mathrm{U}} \cos \chi \\
\sqrt{m_{\mathrm{E}}^{2}+m_{\mathrm{N}}^{2}}
\end{array}\right]=\left[\begin{array}{l}
s_{\mathrm{E}} \\
s_{\mathrm{N}} \\
s_{\mathrm{U}}
\end{array}\right],
\end{gathered}
$$

where $\chi$ is the angle between axes D and E, $\alpha_{\mathrm{I}}$ is the azimuth of the inclination and $\alpha_{\mathrm{D}}$ is the azimuth of declination directions, $\omega$ is the inclination (or zenith) angle between the observation plane and the vertical axis $\mathrm{U}$, and finally $\boldsymbol{s}$ is a unit vector along the axis $\mathrm{I}$. The following angles (Fig. 3b)

$$
\begin{aligned}
& \beta=\sin ^{-1}\left|s \times s_{a}\right| \\
& \gamma=\sin ^{-1}\left|s \times s_{d}\right|
\end{aligned}
$$

should satisfy the control equation: $\delta=\beta+\gamma$.

After the geometric parameters of the observation plane have been derived, the displacements can be computed. According to Fig. 4 the intersection of the two lines

$$
\begin{aligned}
& I=f_{a}(D)=\frac{\Delta S_{a}}{\cos \beta}+D \tan \beta \\
& I=f_{d}(D)=\frac{\Delta S_{d}}{\cos \gamma}-D \tan \gamma
\end{aligned}
$$

have to be solved, where the first terms of right hand sides are the intersections with axis I. They can be simplified as

$$
\begin{aligned}
& \Delta S_{a}=I \cos \beta-D \sin \beta, \\
& \Delta S_{d}=I \cos \gamma+D \sin \gamma .
\end{aligned}
$$

There are several solutions of the two equations, e.g. 


$$
\begin{aligned}
I & =\left(\frac{\Delta S_{d}}{\sin \gamma}+\frac{\Delta S_{a}}{\sin \beta}\right) /(\operatorname{ctg} \beta+\operatorname{ctg} \gamma), \\
D & =\left(\frac{\Delta S_{d}}{\cos \gamma}-\frac{\Delta S_{a}}{\cos \beta}\right) /(\tan \beta+\tan \gamma) .
\end{aligned}
$$

Another solution will be presented in next section.

The following projections

$$
\begin{aligned}
& \Delta U^{\prime}=\frac{I}{\cos \omega}, \\
& \Delta E^{\prime}=\frac{D}{\cos \chi}
\end{aligned}
$$

are different from $\Delta U$ and $\Delta E$ if $\Delta N$ is not zero and the satellite configurations are not symmetric. However, there are some special cases that can be deduced from the introduced equations.

If the satellite configuration is symmetric to the north $\left(\alpha_{a}+\alpha_{d}=2 \pi\right.$ and $\left.\theta_{a}=\theta_{d}\right)$, which leads to $\chi=0$, the following relations can be derived

- if $\Delta N \neq 0$ then $\Delta E^{\prime}=\Delta E$ but $\Delta U^{\prime}$ is biased by $\Delta N$,

- if $\Delta N=0$ then $\Delta E^{\prime}=\Delta E$ and $\Delta U^{\prime}=\Delta U$,

- if $\Delta N=\Delta E=0$ then $\Delta E^{\prime}=0$ and $\Delta U^{\prime}=\Delta U$,

- if $\Delta U=\Delta E=0$ then $\Delta E^{\prime}=0$ and $\Delta U^{\prime}=-\left(\Delta N \cos \alpha_{a} \sin \theta_{a}\right) /(\cos \beta \cos \omega)$.

Both incidence asymmetry $\left(\alpha_{a}+\alpha_{d}=2 \pi\right.$ but $\left.\theta_{a} \neq \theta_{d}\right)$ and azimuth asymmetry $\left(\alpha_{a}+\alpha_{d} \neq\right.$ $2 \pi$ but $\theta_{a}=\theta_{d}$ ) lead to $\chi \neq 0$ and the relations are

- if $\Delta N=0$ then $\Delta E^{\prime}=\Delta E$ but $\Delta U^{\prime}$ is biased by $\Delta E$,

- if $\Delta N=\Delta E=0$ then $\Delta E^{\prime}=0$ and $\Delta U^{\prime}=\Delta U$.

Additionally, the smaller the $\theta_{a}$ and $\theta_{d}$ are, the smaller the $\omega$ is, and the results are more sensitive to $\Delta U$ and $\Delta E$ then to $\Delta N$. 


\subsection{Single data combinations using the Gauss-Markov model}

Supposing two different SAR satellites with ascending and descending LOS changes the following general equations can be written according to Eq. (5)

$$
\left[\begin{array}{l}
\Delta S_{a_{1}} \\
\Delta S_{d_{2}} \\
\Delta S_{a_{3}} \\
\Delta S_{d_{4}}
\end{array}\right]=\left[\begin{array}{lll}
-\sin \alpha_{a_{1}} \sin \theta_{a_{1}} & -\cos \alpha_{a_{1}} \sin \theta_{a_{1}} & \cos \theta_{a_{1}} \\
-\sin \alpha_{d_{2}} \sin \theta_{d_{2}} & -\cos \alpha_{d_{2}} \sin \theta_{d_{2}} & \cos \theta_{d_{2}} \\
-\sin \alpha_{a_{3}} \sin \theta_{a_{3}} & -\cos \alpha_{a_{3}} \sin \theta_{a_{3}} & \cos \theta_{a_{3}} \\
-\sin \alpha_{d_{4}} \sin \theta_{d_{4}} & -\cos \alpha_{d_{4}} \sin \theta_{d_{4}} & \cos \theta_{a_{4}}
\end{array}\right]\left[\begin{array}{c}
\Delta E \\
\Delta N \\
\Delta U
\end{array}\right]
$$

In matrix notation

$$
y=A x
$$

where $\boldsymbol{A}$ is a liner coefficient matrix. If normally distributed unknown correction (or noise) vector $\boldsymbol{v}$ is added to the LOS measurements, the correction equation is

$$
v=A x-y
$$

and the statistical properties of the Gauss-Markov model are

$$
\begin{aligned}
& E\langle\boldsymbol{v}\rangle=0, \\
& D\langle\boldsymbol{v}\rangle=D\langle\boldsymbol{y}\rangle=\boldsymbol{Q}=\sigma_{0}^{2} \boldsymbol{P}^{-\mathbf{1}},
\end{aligned}
$$

where operator $E\langle$.$\rangle stands for expectation and D\langle$.$\rangle for dispersion, \boldsymbol{Q}$ is a known variance matrix, $\sigma_{0}$ is an "a priori" standard deviation of unit weight, which can be chosen arbitrary, and $\boldsymbol{P}$ is a weight matrix. The diagonal elements of variance matrix contain the square of measurements standard deviations as the measure of dispersion. The off-diagonal elements may contain the covariance values.

If $\boldsymbol{A}$ has no rank deficit the solution can be derived by least square adjustment

$$
\begin{aligned}
\hat{\boldsymbol{x}} & =\left(\boldsymbol{A}^{t} \boldsymbol{P} \boldsymbol{A}\right)^{-1}\left(\boldsymbol{A}^{t} \boldsymbol{P} \boldsymbol{y}\right), \\
\widehat{\boldsymbol{v}} & =\boldsymbol{A} \widehat{\boldsymbol{x}}-\boldsymbol{y}, \\
\hat{\sigma}_{0}^{2} & =\frac{\widehat{\boldsymbol{v}}^{t} \boldsymbol{P} \hat{\boldsymbol{v}}}{m-u}, \\
\boldsymbol{Q}_{\hat{x}} & =\hat{\sigma}_{0}^{2}\left(\boldsymbol{A}^{t} \boldsymbol{P} \boldsymbol{A}\right)^{-1},
\end{aligned}
$$


where $t$ signs the transpose matrix, $\hat{\sigma}_{0}^{2}$ is the "a posterior" standard deviation of unit weight, $\boldsymbol{Q}_{\hat{x}}$ is a variance matrix of the estimated unknowns, $m$ is the number of measurements and $u$ is the number of unknowns. In the case when there is no redundancy $(m=u)$, the precision of derived displacements can be estimated by "a priory" data

$$
\boldsymbol{Q}_{\hat{x}}=\sigma_{0}^{2}\left(\boldsymbol{A}^{t} \boldsymbol{P} \boldsymbol{A}\right)^{-1}
$$

If ellipsoidal height changes are available from levelling measurement (or from other estimations) or all the three displacement components are available from GNSS measurements (or from other estimations), the following equations can be written

$$
\begin{aligned}
& {\left[\begin{array}{l}
\Delta S_{a_{1}} \\
\Delta S_{d_{2}} \\
\Delta U_{l}
\end{array}\right]=\left[\begin{array}{ccc}
-\sin \alpha_{a_{1}} \sin \theta_{a_{1}}-\cos \alpha_{a_{1}} \sin \theta_{a_{1}} & \cos \theta_{a_{1}} \\
-\sin \alpha_{d_{2}} \sin \theta_{d_{2}}-\cos \alpha_{d_{2}} \sin \theta_{d_{2}} & \cos \theta_{d_{2}} \\
0 & 0 & 1
\end{array}\right]\left[\begin{array}{l}
\Delta E \\
\Delta N \\
\Delta U
\end{array}\right],} \\
& {\left[\begin{array}{c}
\Delta S_{a_{1}} \\
\Delta S_{d_{2}} \\
\Delta E_{g} \\
\Delta N_{g} \\
\Delta U_{g}
\end{array}\right]=\left[\begin{array}{ccc}
-\sin \alpha_{a_{1}} \sin \theta_{a_{1}}-\cos \alpha_{a_{1}} \sin \theta_{a_{1}} \cos \theta_{a_{1}} \\
-\sin \alpha_{d_{2}} \sin \theta_{d_{2}}-\cos \alpha_{d_{2}} \sin \theta_{d_{2}} & \cos \theta_{d_{2}} \\
1 & 0 & 0 \\
0 & 1 & 0 \\
0 & 0 & 1
\end{array}\right]\left[\begin{array}{l}
\Delta E \\
\Delta N \\
\Delta U
\end{array}\right],}
\end{aligned}
$$

where $l$ subscript indicates the levelling and $g$ the GNSS origin. These observation equations can also be solved by least square adjustment.

In the case of additionally known height changes, Eq. (35) can be rearranged as

$$
\left[\begin{array}{l}
\Delta S_{a_{1}}-\cos \theta_{a_{1}} \Delta U_{l} \\
\Delta S_{d_{2}}-\cos \theta_{d_{2}} \Delta U_{l}
\end{array}\right]=\left[\begin{array}{ll}
-\sin \alpha_{a_{1}} \sin \theta_{a_{1}} & -\cos \alpha_{a_{1}} \sin \theta_{a_{1}} \\
-\sin \alpha_{d_{2}} \sin \theta_{d_{2}} & -\cos \alpha_{d_{2}} \sin \theta_{d_{2}}
\end{array}\right]\left[\begin{array}{l}
\Delta E \\
\Delta N
\end{array}\right]
$$

where the number of unknowns are reduced. If the satellite configuration is symmetric to the north, the number of unknowns cannot be reduced to $\Delta E$ and $\Delta N$, because the reduced $\boldsymbol{A}$ matrix will have a rank deficit as the consequence of satellite symmetry.

The unambiguous inclination and declination components of ascending and descending changes can also be estimated by least square adjustment rearranging Eqs. (19) and (20) as

$$
\left[\begin{array}{l}
\Delta S_{a} \\
\Delta S_{d}
\end{array}\right]=\left[\begin{array}{rr}
\cos \beta & -\sin \beta \\
\cos \gamma & \sin \gamma
\end{array}\right]\left[\begin{array}{l}
I \\
D
\end{array}\right]
$$


Diagonal elements of $\boldsymbol{Q}_{\hat{x}}$ matrices contain the square of the estimated standard deviations $\left(\sigma_{\hat{E}}\right.$, $\left.\sigma_{\widehat{N}}, \sigma_{\widehat{U}}\right)$ which are the measure of precision. Neglecting the standard deviation of unit weight the square roots of the diagonal elements are the measure of geometric dilution of precisions $\left(D O P_{\widehat{E}}, D O P_{\widehat{N}}, D O P_{\widehat{U}}\right)$ and the correlation matrix can also be computed

$$
\boldsymbol{C}(i, j)=\boldsymbol{Q}_{\hat{x}}(i, j) / \boldsymbol{Q}_{\hat{x}}(i, i) / \boldsymbol{Q}_{\hat{x}}(j, j),
$$

where $i, j$ denote the row and column of the matrix elements. The DOP and $C$ values can be computed even if there are no real or redundant measurements.

\section{Results of test computations}

\subsection{Source of test data}

Although, the Sentinel-1 mission of the European Space Agency (ESA) is not yet declared as fully operational, useful Sentinel-1A SAR SLC images are available from 03.10.2014. The used geometric parameters are derived from the position of integrated benchmark established in the Széchenyi István Geophysical Observatory and Sentinel-1A orbit data of two ascending and one descending SAR acquisition series. Their geometric parameters, together with fictional supplementary "IDEAL" satellite are summarized in Table 1. The mean position angels and their standard deviations show a very stable Sentinel-1A satellite orbit.

The displacements of test computations $(\Delta N=-0.02, \Delta E=0.03$ and $\Delta U=-0.15 \mathrm{~m})$ are chosen according to the characteristic values of landslides along the bank of river Danube in Hungary (Újvari et al., 2009; Bányai et al., 2014).

\subsection{Ascending and descending data processing}

According to Section 2.1 only two components ( $I$ and $D)$ can be derived - without any further assumptions - from one ascending and one descending LOS changes. From the available 
Sentinel-1A image series, two observation planes can be selected. Their characteristic angles are given in Table 2 .

Since there are two changes and two unknowns, moreover the equations (21 and 22) are difficult referring to six starting parameters; instead of error propagation the Monte-Carlo simulation was applied to investigate the precision of the estimated values. Some 1000 normally distributed random angles $\left(\alpha_{a}, \theta_{a}\right.$ and $\left.\alpha_{d}, \theta_{d}\right)$ and $\operatorname{LOS}\left(\Delta S_{a}\right.$ and $\left.\Delta S_{d}\right)$ errors were simulated with $\sigma=1$ degree for the positional angles and $\sigma=2 \mathrm{~mm}$ for LOS changes.

The results are given in Table 3. It can be seen that the observation planes are not symmetric at all; consequently, the estimated parameters are biased by the north component, especially the up components.

The observation equation (38) and the Gauss-Markov model of least square adjustment can be used also to determine the unknowns, which are exactly the same as the results of MonteCarlo simulation, despite only the dispersions of LOS changes were taken into account. It means the precision of the estimation is not sensitive to the random errors of positional directions.

The statistics of $A(1)$ - D observation plane

$$
\begin{gathered}
\sigma_{D}=0.0021 \mathrm{~m}, \quad \mathrm{DOP}_{D}=1.1 \\
\sigma_{I}=0.0019 \mathrm{~m}, \quad D_{I}=1.0
\end{gathered},
$$

and for $\mathrm{A}(2)$ - D observation plane

$$
\begin{gathered}
\sigma_{D}=0.0023 \mathrm{~m}, \quad \text { DOP }=1.1 \\
\sigma_{I}=0.0018 \mathrm{~m}, \quad \text { DOP }=0.9 \\
\boldsymbol{C}=\left[\begin{array}{cc}
1 & -0.02 \\
-0.02 & 1
\end{array}\right]
\end{gathered}
$$

are very similar. The small correlations are the consequence of asymmetric satellite geometry. 


\subsection{Single PS estimations with different data fusion}

During the test computations it is supposed that all the observations (LOS, GNSS and levelling) are uncorrelated and their known standard deviations are uniformly $2 \mathrm{~mm}$. Choosing $\sigma_{0}=0.002$ the weight matrices became unit matrices and the solution became simpler. Since we have no real observations Eq. (34) is used for the statistical investigations. The element of Eq. (25) are computed from the parameters of Sentinel (A(2), D) frames and IDEAL satellites (Table 1). The statistical results are

$$
\begin{aligned}
& \sigma_{\widehat{E}}=0.0023 \mathrm{~m}, \quad D_{\widehat{U}} O P_{\widehat{E}}=1.1 \\
& \sigma_{\widehat{N}}=0.0028 \mathrm{~m}, \quad D_{\widehat{N}}=1.4 \\
& \sigma_{\widehat{U}}=0.0016 \mathrm{~m}, \quad \mathrm{DOP}_{\widehat{U}}=1.6 \\
& \boldsymbol{C}=\left[\begin{array}{ccc}
1 & 0.02 & -0.01 \\
0.02 & 1 & -0.57 \\
-0.01 & -0.57 & 1
\end{array}\right] .
\end{aligned}
$$

This would be one of the ideal solutions which apply only InSAR data. If the parameters of the three available Sentinel-1A frames $(\mathrm{A}(1), \mathrm{A}(2)$ and $\mathrm{D})$ are used, the statistics

$$
\begin{aligned}
& \sigma_{\hat{E}}=0.0019 \mathrm{~m}, \quad D O P_{\widehat{E}}=1.0 \\
& \sigma_{\widehat{N}}=0.1749 \mathrm{~m}, D O P_{\widehat{N}}=87.4 \text {, } \\
& \sigma_{\widehat{U}}=0.0252 \mathrm{~m}, \quad D O P_{\widehat{U}}=12.6 \\
& \boldsymbol{C}=\left[\begin{array}{ccc}
1 & -0.11 & -0.09 \\
-0.11 & 1 & 1.00 \\
-0.09 & 1.00 & 1
\end{array}\right]
\end{aligned}
$$

show the very poor condition of this solution.

In the next investigations the Sentinel-1A frames $(A(2), D)$ are combined with levelled changes. The Eq. (35) leads to the statistics

$$
\begin{aligned}
& \sigma_{\widehat{E}}=0.0023 \mathrm{~m}, D O P_{\hat{E}}=1.2 \\
& \sigma_{\widehat{N}}=0.0194 m, D O P_{\widehat{N}}=9.7 \text {, } \\
& \sigma_{\widehat{U}}=0.0020 \mathrm{~m}, D O P_{\widehat{U}}=1.0 \\
& \boldsymbol{C}=\left[\begin{array}{ccc}
1 & 0.06 & 0.04 \\
0.06 & 1 & 0.74 \\
0.04 & 0.74 & 1
\end{array}\right]
\end{aligned}
$$

while Eq.(37) to: 


$$
\begin{aligned}
& \sigma_{\widehat{E}}=0.0023 \mathrm{~m}, \quad D O P_{\widehat{E}}=1.2 \\
& \sigma_{\widehat{N}}=0.0130 \mathrm{~m}, \quad D O P_{\widehat{N}}=6.5 \text { ' } \\
& \boldsymbol{C}=\left[\begin{array}{cc}
1 & 0.05 \\
0.05 & 1
\end{array}\right]
\end{aligned}
$$

The errorless treatment of levelled height change does not significantly improve the solution of north component. However, in the case of large north movements, this precision may be acceptable.

Finally the fusion with GNSS derived changes is investigated. The Eq. (36) provides the statistics

$$
\begin{aligned}
& \sigma_{\widehat{E}}=0.0015 \mathrm{~m}, \quad D O P_{\widehat{E}}=0.8 \\
& \sigma_{\widehat{N}}=0.0020 m, \quad D O P_{\widehat{N}}=1.0 \text {, } \\
& \sigma_{\widehat{U}}=0.0013 \mathrm{~m}, \quad D O P_{\widehat{U}}=0.7 \\
& \boldsymbol{C}=\left[\begin{array}{ccc}
1 & 0 & -0.01 \\
0 & 1 & 0.11 \\
-0.01 & 0.11 & 1
\end{array}\right]
\end{aligned}
$$

which seems to be an excellent data combination.

\section{Discussion of the test computations}

As it was expected, the available SAR data are not suitable to derive $3 \mathrm{D}$ changes by themselves. A properly chosen complementary satellite would be the solution to provide excellent 3D solutions using only SAR data.

Having one ascending and one descending LOS change, only two components ( $I$ and $D$ ) can be determined unambiguously in the observation plane. The Monte-Carlo simulation proved that the solution is not sensitive to the random errors of positional angles; therefore it is enough to take the standard deviations of changes in LOS directions into account. The estimated precisions are practically the same, as the ideal complementary satellite case would provide, but we have no information about the north component, which can bias both the east and up components in the case of asymmetric satellite configurations. 
In the lack of complementary satellite, additional geodetic data can be used. The fusion even with errorless levelling data cannot significantly improve the estimation of north component. However, the direct fusion of LOS data with GNSS displacements can significantly improve the solution, although the estimation of north component is the direct impact of GNSS derived values. Since the nearly vertical inclination is the best estimable component of ascending and descending PSI data, which is partly biased by north movement, and the height is the weakest estimable component of GNSS technique, they are excellent complementary technologies.

On the whole, the presented statistical investigation can be used only to compare the different possibilities, because in the practice there may be other limitations that were not taken into account during the test computations.

\section{Summary and conclusions}

The geometric information content of ascending and descending LOS data and their equations are derived. One ascending and one descending LOS data of same PS can be used to determine unambiguously only two characteristic components in the observation plane, which can be transformed to east and vertical directions. The north component remains unknown, but usually biases both the east and vertical components.

While there will be no complementary SAR satellite at our disposal and thus autonomous 3D displacement estimation is not provided by this way, the different data fusion with GNSS derived displacement are the best candidates for the 3D procedures.

In the case of geodetic networks using integrated benchmarks, which are not measured continuously by GNSS technique, the ascending and descending LOS data can be utilized between the periods of two GNSS observation campaigns. Taking into account the time series nature of PSI and GNSS data the Kalman filter techniques seems to be one of the optimal methods to update the 3D displacements continuously. 


\section{Acknowledgement}

This study was funded by the Government of Hungary through an ESA Contract (No. 4000114846/15/NL/NDe /15/NL/NDe) under the PECS (Plan for European Cooperating States). The view expressed herein can in no way be taken to reflect the official opinion of the European Space Agency.

\section{References}

Bányai, L., Mentes, Gy., Újvári, G., Kovács, M., Czap, Z., Gribovszki, K., Papp, G., 2014. Recurrent landsliding of a high bank at Dunaszekcső, Hungary: Geodetic deformation monitoring and finite element modeling", Geomorphology 210, 1-13.

Catalao, J., Nico, G., Hanssen, R., Catita, C., 2011. Merging GPS and atmospherically corrected InSAR data to map 3-D terrain displacement velocity. IEEE Transactions on Geoscience and Remote Sensing 49(6), 2354-2360.

Cascini, L., Fornaro, G., Peduto, D., 2010. Advanced low- and full-resolution DInSAR map generation for slow-moving landslide analysis at different scales. Engineering Geology $112,29-42$.

Colesanti, C., Ferretti, A., Prati, C., Rocca, F., 2003. Monitoring landslides and tectonic motions with the Permanent Scatterers Technique. Engineering Geology 68, 3-14.

Garthwaite, M.C., Thankappan, M., Williams, M.L., Nancarrow, S.; Hislop, A., Dawson, J., 2013. Corner reflectors for the Australian Geophysical Observing System and support for calibration of satellite-borne synthetic aperture radars. IGARSS 2013 publication, 266269.

Gray, L., 2011. Using multiple RADARSAT InSAR pairs to estimate a full three-dimensional solution for glacial ice movement. Geophys. Res. Lett. 38, L05502, doi:10.1029/2010GL046484.

Gisinger, C., Balss, U., Pail, R., Zhu, X. X., Montazeri, S., Gernhardt, S., and Eineder, M., 2015. Precise Three-Dimensional Stereo Localization of Corner Reflectors and Persistent Scatterers With TerraSAR-X," IEEE Trans. Geosci. Remote Sens., vol. 53, no. 4, pp. 1782-1802. 
Gudmundsson, S., Gudmundsson, M.T., Björnsson, H., Sigmundsson, F., Rott, H., Carstensen, J.M., 2002. Three-dimensional glacier surface motion maps at the Gjalp eruption site, Iceland, inferred from combining InSAR and other ice-displacement data. Annals of Glaciology 34(1), 315-322.

Guglielmino, F., Nunnari, G., Puglisi, G., Spata, A., 2011. Simultaneous and Integrated Strain Tensor Estimation From Geodetic and Satellite Deformation Measurements to Obtain Three-Dimensional Displacement Maps. IEEE Transactions on Geoscience and Remote Sensing 49(6), 1815-1826.

Hooper, A., Zebker, H., Segall, P., Kampes, B., 2004. A new method for measuring deformation on volcanoes and other natural terrains using InSAR persistent scatterers. Geophysical Research Letters 31, L23611.

Hooper, A., 2008. A multi-temporal InSAR method incorporating both persistent scatterer and small baseline approaches. Geophysical Research Letters, 35 (16),

Hooper, A., Bekaert, D., Spaans, K., Arikan, M., 2012. Recent advances in SAR interferometry time series analysis for measuring crustal deformation. Tectonophysics 514-517, 1-13.

Hu, J., Li, Z.W., Ding, X.L., Zhu, J.J., Zhang, L., Sun, Q., 2014. Resolving Threedimensional surface displacements from InSAR measurements: A review. Earth-Science Reviews 133, 1-17.

Hung, W.C., Hwang, C., Chen, Y.A., Chang, C.P., Yen, J.Y., Hooper, A., Yang, C. Y., 2011. Surface deformation from persistent scatters SAR interferometry and fusion with leveling data: A case study over the Choushui River Alluvial Fan, Taiwan. Remote Sensing of Environment 115(4), 957-967.

Kumar, V., Venkataramana, G., Høgda, K.A., 2011. Glacier surface velocity estimation using SAR interferometry technique applying ascending and descending passes in Himalayas. International Journal of Applied Earth Observation and Geoinformation 13(4), 545-551.

Manzo, M., Ricciardi, G.P., Casu, F., Ventura, G., Zeni, G., Borgström, S., Berardino, P., Del Gaudio, C., Lanari, R., 2006. Surface deformation analysis in the Ischia Island (Italy) based on spaceborne radar interferometry. Journal of Vulcanology and Geothermal Research 151(4), 399-416.

Meyer, F.J., McAlpin, D.B., Gong, W., Ajadi O., Arko, S., Webley, P.W., Dehn, J., 2015. Integrating SAR and derived products into operational volcano monitoring and decision support systems. ISPRS Journal of Photogrammetry and Remote Sensing 100, 106-117. 
Plank, S., Singer, J., Thuro, K., 2013. Assessment of number and distribution of persistent scatterers prior to radar acquisition using open access land cover and topographical data. ISPRS Journal of Photogrammetry and Remote Sensing 85, 132-147.

Riddick, S.N., Schmidt, D.A., Deligne, N.I., 2012. An analysis of terrain properties and the location of surface scatterers from persistent scatterer interferometry. ISPRS Journal of Photogrammetry and Remote Sensing 73, 50-57.

Rocca, F., 2003. 3D motion recovery from multi-angle and/or left right interferometry. Proceedings of the Third International Workshop on ERS SAR.

Samsonov, S., Tiampo, K., 2006. Analytical Optimization of a DInSAR and GPS Dataset for Derivation of Three-Dimensional Surface Motion. IEEE Geoscience and Remote Sensing Letters 3(1), 107-111.

Samsonov, S., Tiampo, K., Rundle, J., Li, Z., 2007. Application of DInSAR-GPS Optimization for Derivations of Fine-Scale Surface Motion Maps of Southern California. IEEE Transactions on Geoscience and Remote Sensing 45(2), 512-521.

Tralli, D.M., Blom, R.G., Zlotnicki, V., Donnellan, A., Evans, D.L., 2005. Satellite remote sensing of earthquake, volcano, flood, landslide and coastal inundation hazards, ISPRS Journal of Photogrammetry \& Remote Sensing 59, 185-198.

Újvári, G., Mentes, Gy., Bányai, L., Kraft, J., Gyimóthy, A., Kovács, J., 2009. Evolution of a bank failure along the River Danube at Dunaszekcső, Hungary. Geomorphology 109, 197-209.

Wasowski, J., Bovenga, F., 2014. Investigating landslides and unstable slopes with satellite Multi Temporal Interferometry: Current issues and future perspectives. Engineering Geology 174, 103-138.

Wright, T.J., Parsons, B.E., Lu, Z., 2004. Toward mapping surface deformation in three dimensions using InSAR. Geophys. Res. Lett. 31, L01607, doi:10.1029/2003GL018827. 


\section{Figures}

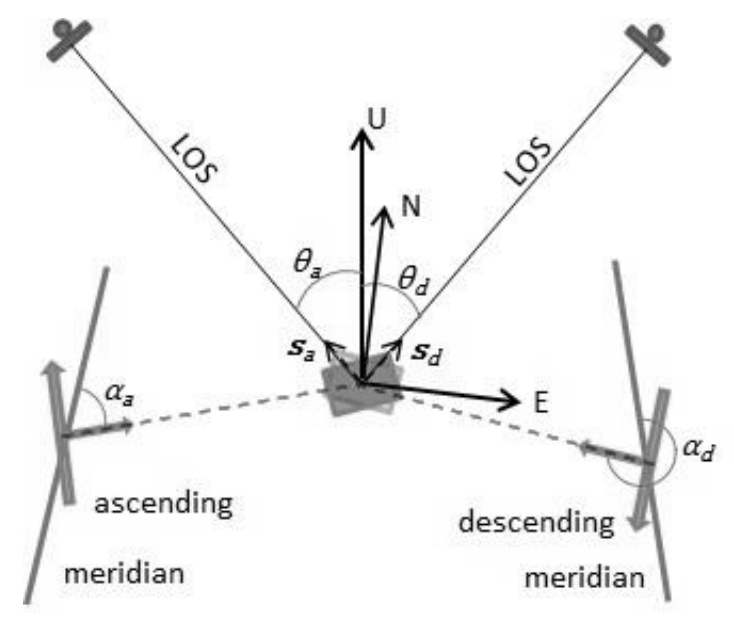

Fig. 1. Geometry of ascending and descending LOS observations of the same resolution cell on the Earth surface. $\alpha_{a}$ and $\alpha_{d}$ are the LOS azimuths, $\theta_{a}$ and $\theta_{d}$ are the incidence angles of the SAR illuminations, $\boldsymbol{s}_{a}$ and $\boldsymbol{s}_{d}$ are the unit vectors of ascending and descending satellite directions given in the ellipsoidal topocentric coordinate system, $U$ is the direction of the ellipsoidal normal (up), $\mathrm{N}$ is a direction to the north and $\mathrm{E}$ is the direction to the east in the plane perpendicular to $\mathrm{U}$. 

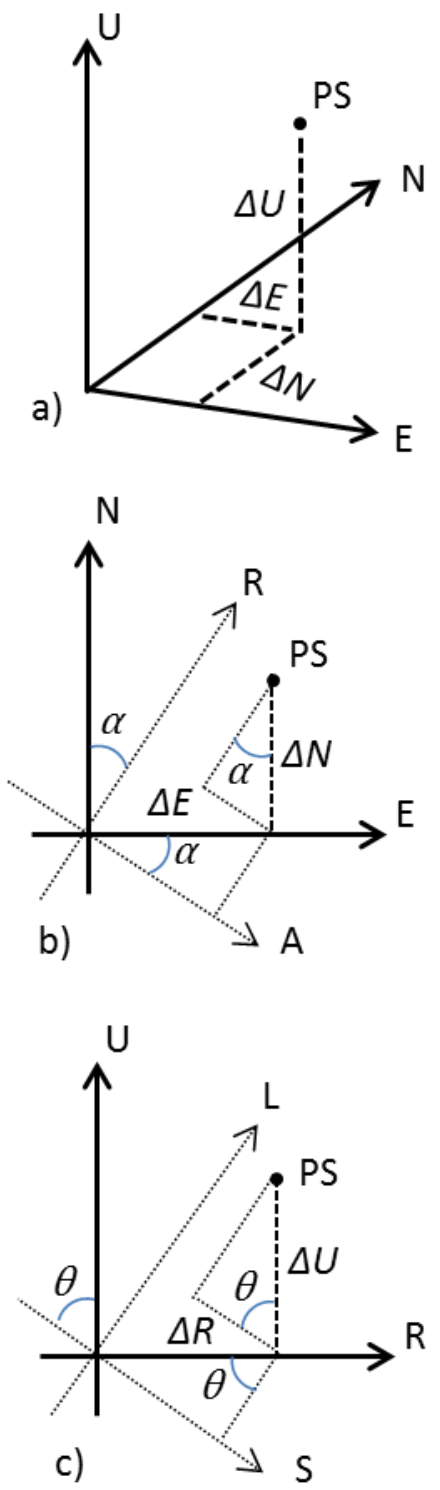

Fig. 2. Displacements of the PS in ellipsoidal topocentric coordinate system (E, N, U). a) PS is displaced by $\Delta E, \Delta N$ and $\Delta U$ values. b) $\mathrm{R}$ and $\mathrm{A}$ axes are connected to $\alpha$ LOS azimuth in the N-E plane. c) $\mathrm{S}$ and $\mathrm{L}$ axes are connected to $\theta$ incidence angle in the U-R plane. 


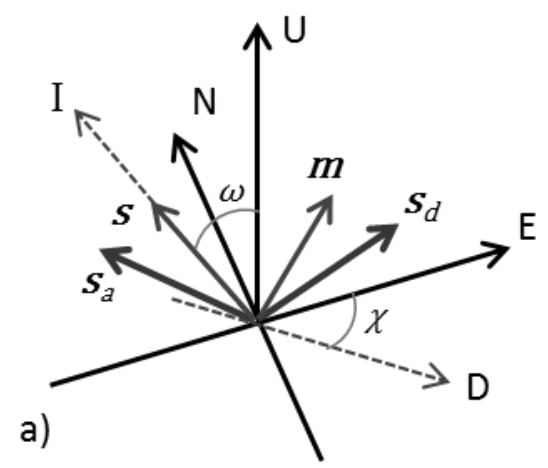

b)

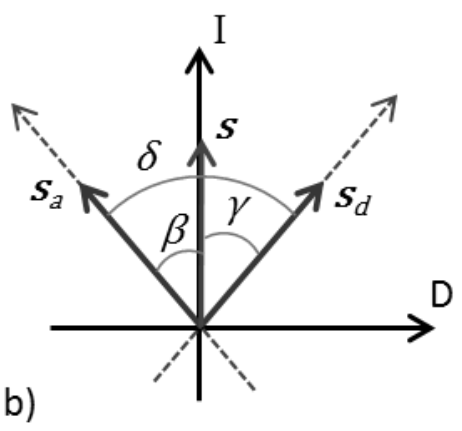

Fig.3. Geometric parameters of the observation plane defined by $\boldsymbol{s}_{a}$ and $\boldsymbol{s}_{d}$ unit vectors in ellipsoidal topocentric coordinate system $(\mathrm{E}, \mathrm{N}, \mathrm{U})$. a) $\boldsymbol{m}$ is a normal unit vector of the plane, I and $\mathrm{D}$ are the inclination and declination axes, $\omega$ and $\chi$ are the inclination and declination angles, $\boldsymbol{s}$ is the unit vector along I. b) $\boldsymbol{s}_{a}, \boldsymbol{s}$ and $\boldsymbol{s}_{d}$ are the unit vectors in the I-D plane, $\delta, \beta$ and $\gamma$ are the angels between the unit vectors.

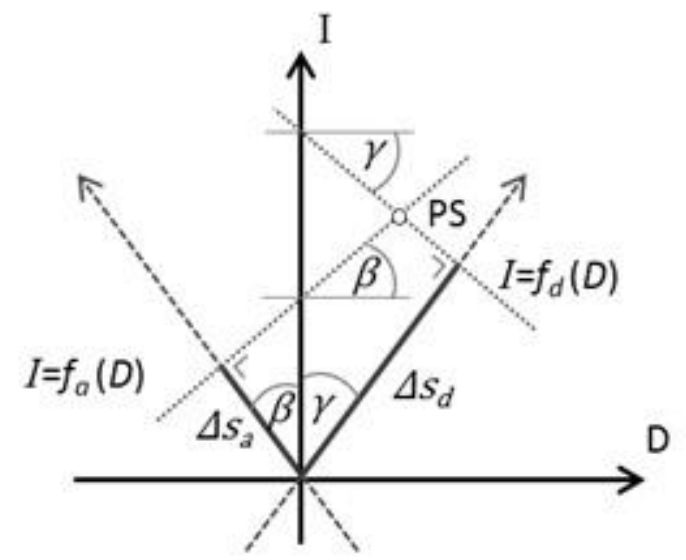

Fig. 4. Position of the PS in the D-I plane, where $\Delta S_{a}$ and $\Delta S_{d}$ are the displacements towards the satellite positions. The intersection of the lines $I=f_{a}(D)$ and $I=f_{d}(D)$, which are perpendicular to the displacements, defines the position of the PS. 


\section{Tables}

Table 1. Ascending (A) and descending (D) geometry of Sentinel-1A and IDEAL acquisitions. $N r$ is the number of available images, $\alpha$ is the mean LOS azimuth, $\theta$ is the mean LOS incidence angle, $\sigma$ is their standard deviations and $\Delta S$ is the simulated LOS change.

\begin{tabular}{cccccccc}
\hline \multicolumn{2}{c}{ Satellite } & $N r$ & $\alpha$ & $\sigma_{\alpha}$ & $\theta$ & $\sigma_{\theta}$ & $\Delta S(\mathrm{~m})$ \\
\hline S-1A & $\mathrm{A}(1)$ & 12 & $81^{\circ} 08^{\prime} 04^{\prime \prime}$ & $2 "$ & $45^{\circ} 21^{\prime} 02^{\prime \prime}$ & $11^{\prime \prime}$ & -0.1243 \\
S-1A & $\mathrm{A}(2)$ & 8 & $79^{\circ} 37^{\prime} 12^{\prime \prime}$ & $2 "$ & $36^{\circ} 41^{\prime} 25^{\prime \prime}$ & $14^{\prime \prime}$ & -0.1358 \\
S-1A & $\mathrm{D}$ & 5 & $279^{\circ} 46^{\prime} 30^{\prime \prime}$ & $2^{\prime \prime}$ & $40^{\circ} 20^{\prime} 03^{\prime \prime}$ & $16^{\prime \prime}$ & -0.0930 \\
\hline IDEAL & $\mathrm{A}$ & - & $169^{\circ}$ & - & $37^{\circ}$ & - & - \\
IDEAL & $\mathrm{D}$ & - & $189^{\circ}$ & - & $40^{\circ}$ & - & - \\
\hline
\end{tabular}

Table 2. Characteristic angles of Sentinel-1A observation planes in degree.

\begin{tabular}{cccccc}
\hline Obs. plain & $\beta$ & $\gamma$ & $\omega$ & $\alpha_{D}$ & $\alpha_{I}$ \\
\hline $\mathrm{A}(1)-\mathrm{D}$ & 44.72 & 39.58 & 8.51 & 89.63 & 179.63 \\
$\mathrm{~A}(2)-\mathrm{D}$ & 35.95 & 39.68 & 7.91 & 90.36 & 180.36 \\
\hline
\end{tabular}

Table 3. Displacements derived in the observation planes in meter.

The $\sigma$ standard deviations are estimated by Monte-Carlo simulations.

\begin{tabular}{ccccccc}
\hline Obs. plain & $D$ & $\sigma_{D}$ & $I$ & $\sigma_{I}$ & $\Delta E^{\prime}$ & $\Delta U^{\prime}$ \\
\hline $\mathrm{A}(1)-\mathrm{D}$ & 0.0299 & 0.0021 & -0.1454 & 0.0020 & 0.0299 & -0.1470 \\
$\mathrm{~A}(2)-\mathrm{D}$ & 0.0301 & 0.0023 & -0.1458 & 0.0018 & 0.0301 & -0.1472 \\
\hline & & & & known: & 0.0300 & -0.1500 \\
\hline
\end{tabular}

